
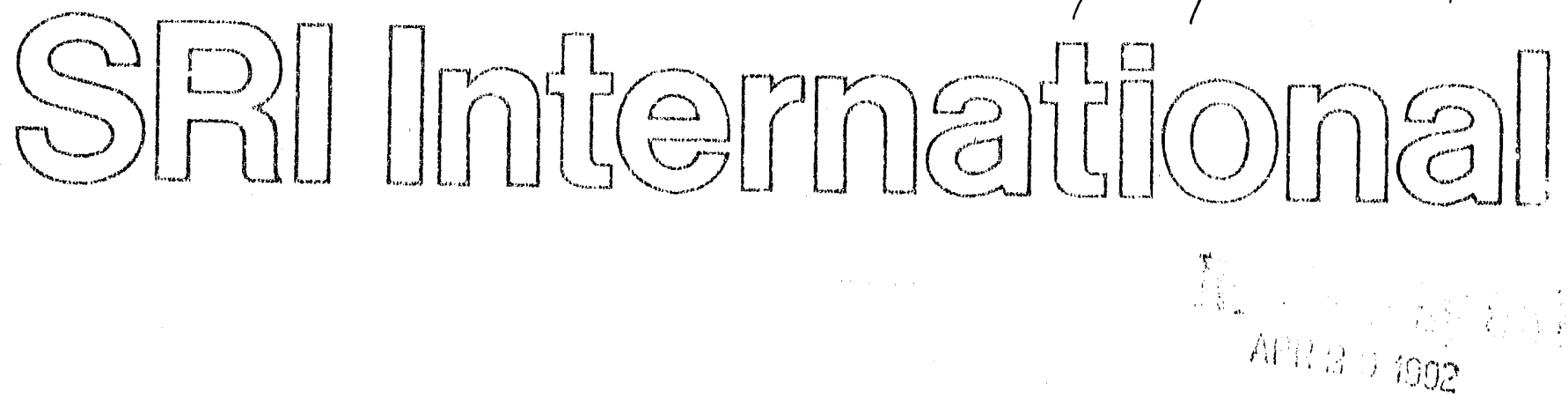

22 January 1991

\title{
COMBUSTION RESEARCH PROGRAM: FLAME STUDIES, LASER DIAGNOSTICS, AND CHEMICAL KINETICS
}

\author{
PART I: Progress Report \\ Covering the Period 15 July 1987 through 3 December 1990
}

Continuation Proposal PYU 91-012

Prepared by:

David R. Crosley, Associate Director

Molecular Physics Laboratory

Prepared for:

Department of Energy

Office of Energy Research

Division of Chemical Sciences

Germantown, MD 20545

Attn: Dr. A. H. Laufer, ER 141

SRI Project No. 2477

Grant No. DE-FG03-86ER1357I

Approved:

DISCLAIMER

Donald J. Eckstrom, Director This report was prepared as an account of work sponsored by an agency of the United States Molecular Physics Laboratory employees, Neither the United States Government nor any agency thereof, nor any of their bility for the accuracy warranty, express or implied, or assumes any legal liability or responsi-

G. R. Abrahamson

Senior Vice President

Physical Sciences Division process disclosed, or reforest, or process disclosed, or represents that its use would not infringe privately owned rights. Refermanufacturer, or specific commercial produci, process, or service by trade name, trademark, mendation, or favoring by thes not necessarily constitute or imply its endorsement, recomand opinions of authors expressed hed States Government or any agency thereof. The views United States Government or any agency thereof. 
DOE/ER/13571--TI

PROGRESS

DE92 012575

\section{OVERVIEW}

During the past four years, we have devoted considerable effort to the quantitative detection of hydride radicals in flames, including a detailed study of the care that must be taken to correctly measure $\mathrm{OH}$ radical concentrations in flames. A large part of these studies has concerned collisional quenching of hydride radical species $(\mathrm{OH}, \mathrm{NH}$, and $\mathrm{NH}_{2}$ ), in particular the dependence upon rotational level and collision velocity (temperature). The results on $\mathrm{OH}$ and $\mathrm{NH}$ have shown unique and interesting behavior from the viewpoint of fundamental molecular dynamics, pointing to quenching often governed by collisions on an anisotropic, attractive surface, whereas $\mathrm{NH}_{2}$ quenching appears to depend on state-mixing considerations, not dynamic control. This state-specific behavior of these small, ti eoretically tractable hydrides has direct ramifications for quantitative flame diagnostics. Our other effort in the diagnostic area has been repeated but unsuccessful searches for LIF in the vinyl radical. The reaction kinetics studies have included three topics of current controversy. The first is an examination, via RRKM and transition state theory calculations, of recent, conflicting results for high temperature rate constants for $\mathrm{OH}+\mathrm{C}_{2} \mathrm{H}_{4}$. The second is a remeasurement, using combined LP/LF and laser pyrolysis/resonance-enhanced multiphoton ionization (REMPI) detection, of the key $\mathrm{CH}_{3}+\mathrm{O}_{2}$ reaction rate constant, which was thought to be low according to earlier measurements in our laboratory with a very low pressure photolysis (VLPФ) apparatus but was suggested to occur by recent experiments elsewhere. The third topic is RRKM calculations on reactions of alkyl radicals and hydrogen halides, because of a controversy between results from our laboratory and that of Gutman, with serious implications for combustion chemistry.

The part of this research emphasizing flame studies is a deviation from the work proposed four years ago, in which the planned LIF development included spectroscopic studies of $\mathrm{NH}_{3}, \mathrm{HCCO}$, and vinyl radicals and quenching measurements on $\mathrm{NH}_{2}$ and quenching of high rotational levels in NH. Our awareness of the need for accurate flame $\mathrm{OH}$ and temperature measurements prompted this substitution. In Part II of this proposal, which describes the proposed research, we include the work on $\mathrm{NH}_{3}$ detection that was not performed under the current grant. 


\section{QUANTITATIVE LIF FLAME DIAGNOSTICS}

A stringent test of the predictions from detailed computer models of flame chemistry is the profile of reactive intermediates ( $\mathrm{OH}$ and other radicals) and the profile $0^{2}$ the temperature (the latter profile is often critical input to the model calculation). Although qualitative insight can oe obtained from relative profiles, even regions of appearance and disappearance of certain species, it is necessary to have accurate results for $\mathrm{OH}$ (a marker of the $\mathrm{O} / \mathrm{H}$ radical pool) and the temperature.

Our experience modelling flames near 10 Torr on our low pressure burner has taught us that such highly spatially resolved flames indeed furnish critical comparisons but that considerable care must be taken to obtain results accurate enough to be meaningfully compared. Two distinct albeit closely related issues were investigated: (1) the accurate determination of $\mathrm{OH}$ radical concentrations, and (2) the accurate determination of a spatially resolved temperature profile. Many subtle effects producing systematic errors can occur, and these can be unnoticed if the only criterion used is statistical goodness of fit.

The experiments were performed in $\mathrm{H}_{2} / \mathrm{N}_{2} \mathrm{O}$ and $\mathrm{H}_{2} / \mathrm{O}_{2}$ flames burning at 7.2 Torr pressure, on a 6-cm-diameter porous plug burner operated in an evacuated chamber. An excimer-laser-pumped dye laser of 10-ns pulse length duration excited the $\mathrm{OH}$ in the $(0,0)$ band of the $\mathrm{A}^{2} \Sigma^{+}-\mathrm{X}^{2} \Pi_{\mathrm{i}}$ system, and fluorescence in this same band was monitored through a 0.3-m monochromator. The resulting photomultiplier signal was fed to a transient digitizer for time decay determinations or to a boxcar integrator for temperature and concentration measurements.

To perform quantitative comparisons between flame measurements and model calculations of combustion chemistry, it is crucial to have accurate temperature measurements that are precisely spatially correlated with the species determinations. This precision is crucial because of the need to relate number density in a particular level to mo.'e fraction and especially because of the highly nonlinear effect of temperature on a chemical reaction sequence. A systematic error of 100 or $200 \mathrm{~K}$ could render meaningless such comparison with a model. Flame temperatures were determined by computer-controlled rotational excitation scans in the R-branch region of the $\mathrm{OH}(\mathrm{A}-\mathrm{X})$ 0-0 band; a prompt and narrow boxcar gate ensures that little rotational relaxation has occurred. The spectra are fit directly to two parameters: a linewidth and a temperature. This technique has been routinely applied to broadband CARS temperature measurements but has been used only once previous! $y^{8}$ for LIF. Precise fits are obtained, with $<3 \%$ errors $(2 \sigma)$ as gauged by 
replicate scans ove a period of months. However, systematic errors are more important for accurate measurements. These errors are due to spectral biasing ${ }^{9}$ against fluorescence from high $\mathrm{N}^{\prime}$; the choice of gate delay, which is important because higher $\mathrm{N}^{\prime}$ both are quenched and radiate more slowly; and flame optical depth with absorption $>5 \%$.

For flame measurements at atmospheric pressure, particularly where twodimensional imaging is desired, knowledge of the rate of collisional quenching is needed to determine the fluorescence quantum yield. Previous measurements in this laboratory have shown that values for quenching cross sections, $\mho_{\mathrm{Q}}$, for $\mathrm{A}^{2} \Sigma^{+} \mathrm{OH}$ are large and vary with collider, $\mathbf{N}$, and temperature. The total quenching can probably be estimated ${ }^{10}$ to within $30 \%$ if the fuel/oxidarit composition and temperature are known. Here, we measured quenching rates directly in $\mathrm{H}_{2} / \mathrm{N}_{2} \mathrm{O}$ flames. In the burnt gases, there is a slight variation with $N^{\prime}$; in the flame zone, the quantum yield dropped by $25 \%$, increasing from $N^{\prime}=3$ to $\mathrm{N}^{\prime}=8$. There was a $15 \%$ variation for mixing ratios between 0.8 and 1.5 .

Spatial concentration profiles of $\mathrm{OH}$ were measured in the $\mathrm{H}_{2} / \mathrm{O}_{2}$ and $\mathrm{H}_{2} / \mathrm{N}_{2} \mathrm{O}$ flames, and $\mathrm{NH}$ was also measured in the latter flame. These profiles were compared with results from a computer model that was assembled using the Sandia flame code PREMIX ${ }^{11}$ and incorporated our choices of rate constants, to help understand the responsible chemical processes and calculate the mole fractions of water, hydrogen atoms, and free radicals in the mixed oxidant flames (see below).

The $\mathrm{H}_{2} / \mathrm{O}_{2}$ flames at low pressure have an interesting behavior. The three-body chain termination reactions occur very slowly, and the flame does not reach equilibration in the chamber, with a temperature of only $1200 \mathrm{~K}$ many centimeters above the burner surface. Copious amounts of hydrngen atoms are produced, and these diffuse backward into the unburnt gases and decompose the reactants. $\mathrm{OH}$ could be observed within $1 \mathrm{~mm}$ of the burner surface in sufficient quantity that temperature measurements could be performed; there, the temperature was as low as $390 \mathrm{~K}$. We plan to exploit this "warm" flame region in the proposed research.

The $\mathrm{H}_{2} / \mathrm{N}_{2} \mathrm{O}$ flame is terminated by bimolecular reactions and therefore goes much closer to completion, with final temperatures at $2300 \mathrm{~K}$. Flames of intermediate temperatures in the burnt gases were obtained by using mixtures of the oxidants, and quenching measurements were made in these flames. 


\section{QUENCHING OF DIATOMIC HYDRIDES}

Knowledge of the quenching of electronically excited states is necessary to determine the quantum yield for LIF measurements, and accordingly we have spent considerable effort on the quenching of the $\mathrm{OH}$ and $\mathrm{NH}$ radicals. These results have shown an extremely interesting and unique dependence on both rotational level and temperature that is of considerable fundamental molecular dynamic interest. Note that these species are small enough to be fully theoretically tractable, and, spurred by both our collision studies and the recent observation of $\mathrm{OH}$ van der Waals complexes, several groups are commencing computations of the excited state surfaces and trajectory studies of the collision dynamics.

Earlier measurements ${ }^{12}$ on the $\mathrm{A}^{2} \Sigma^{+}, \mathrm{v}^{\prime}=0$ level of $\mathrm{OH}$ in our laboratory had shown that quenching cross sections, $\sigma_{Q}$, are generally large and decrease with increasing rotational level and with temperature. This points to an interaction governed by anisotropic attractive forces. Measurements on the $\mathrm{A}^{3} \Pi_{\mathrm{i}}, \mathrm{v}^{\prime}=0$ level of $\mathrm{NH}$ have been made at elevated temperature in a laser pyrolysis/laser fluorescence system ${ }^{13}$ and at room temperature in a discharge flow system. For most colliders investigated at room temperature, $\sigma_{\mathrm{Q}}(\mathrm{NH})$ was found to decrease with increasing rotational level. The amount of rotational level variation was least for the most efficient quenchers (like $\mathrm{NH}_{3}$, with $\sigma_{Q} \sim 90 \AA^{2}$ ) and more for colliders with smaller quenching cross sections. For many colliders, $\sigma_{\mathrm{Q}}$ decreased with temperature. Again, an anisotropic attractive surface appears to be involved, although a correlation with simple long-range attractive forces calculations is not successful as for $\mathrm{OH}$.

$\mathrm{OH}$ quenching was measured in the burnt gas region of low pressure flames of $\mathrm{H}_{2}$ burning mixtures of $\mathrm{O}_{2}$ and $\mathrm{N}_{2} \mathrm{O}$ at 7.2 Torr. Vyrying the oxidizer mole fraction of $\mathrm{O}_{2}$ from 1 to 0 permitted the temperature to be varied between 1200 and $2300 \mathrm{~K}$, as measured by rotational excitation scans. The burnt gases were composed of a mixture of species, mostly $\mathrm{H}_{2} \mathrm{O}$ and $\mathrm{N}_{2}$ for pure $\mathrm{N}_{2} \mathrm{O}$ and mostly $\mathrm{H}_{2} \mathrm{O}$ and $\mathrm{H}$ for pure $\mathrm{O}_{2}$, as determined by a flame chemistry model. The 10-ns pulse from a dye laser excited the $\mathrm{OH}$, and the fluorescence through a small monochromator was processed by a transient digitizer. Quenching rate constants were determined by subtracting the known radiative rate from the decay rate measured at the fixed pressure. The rotational levels $N^{\prime}=3,8$, and 16 were chosen for the laser excitation. Although some rotational relaxation occurs, most of the population is found in the initially pumped level and the molecules that have relaxed are found in levels near the initially pumped level. Thus, the quenching rate constants 
measured are specific to the initially excited level and permit an examination of the temperature variation of the rotational level dependence of $\sigma_{\mathrm{Q}}$. The results show clearly that the magnitude of the $\mathrm{N}^{\prime}$-dependence of quenching diminishes with increasing temperature: at $300 \mathrm{~K}, \sigma_{\mathrm{Q}}\left(\mathrm{N}^{\prime}=3\right) / \sigma_{\mathrm{Q}}\left(\mathrm{N}^{\prime}=16\right)=1.7 \pm 0.2$, while at $2300 \mathrm{~K}$, the ratio is $1.02 \pm 0.04$, where the room temperature results are from LIF decay measurements ${ }^{14}$ following $\mathrm{OH}$ production by the reaction $\mathrm{O}\left({ }^{1} \mathrm{D}\right)+\mathrm{H}_{2} \mathrm{O}$.

These results on the rotational level dependence of $\sigma_{\mathrm{Q}}$ of $\mathrm{NH}$ (less variation with more efficient colliders) and $\mathrm{OH}$ (less variation at higher temperature) can be explained within a consistent picture. The polar nature of the hydrides leads to long-range potential interaction with the colliders that is attractive and highly anisotropic, having in some orientations deep valleys that promote the formation of a transitory complex which leads to the quenching. These valleys are important for less efficient quenchers. When the radical is not rotating, it and the collider can easily find these valleys and form the complex; as it rotates, however, it averages over the surface during the approach and washes out the influence of these particularly effective regions. For an efficient collider like polar $\mathrm{NH}_{3}$, however, the interactions are so strong that the complex can form readily regardless of approach angle. Then the additional attraction at the preferred orientation adds little, and $\sigma_{\mathrm{Q}}$ is not enhanced for slowly rotating radicals as it is for the inefficient quenchers. As the temperature (collision velocity) increases, the $\sigma_{Q}$ for each $N^{\prime}$ should decrease because of the attractive force influence. However, the $\sigma_{\mathrm{Q}}$ for low $\mathrm{N}^{\prime}$ decreases faster than that for high $N^{\prime}$ because, at a higher collision velocity, the enhancement due to the preferred orientations is inhibited in the same way as it is by faster rotation.

These results raise questions about the variation of $\sigma_{\mathrm{Q}}$ with rotation at very high $\mathrm{N}^{\prime}$, currently under irivestigation (see below).

\section{QUENCHING OF $\mathrm{NH}_{2}$ RADICALS AT ROOM TEMPERATURE}

The ability to observe the rotational level dependence of quenching in $\mathrm{NH}$ and $\mathrm{OH}$ depends on the fact that quenching proceeds faster than rotational thermalization; the latter is relatively slow in these molecules owing to the large level spacing. $\mathrm{NH}_{2}$ radicals are known to quench rapidly even with He collider and therefore also appeared to be a viable candidate for a similar study. Again, $\mathrm{NH}_{2}$ is a small molecule with theoretical tractability, although matters are considerably more complicated here than for the diatomics. A higher energy level density is caused by the added vibrations, and the ground and excited electronic states, the two components of a Renner-Teller pair, are known to perturb one another. $\mathrm{NH}_{2}$ is a key intermediate in the chemistry of formation of $\mathrm{NO}_{\mathrm{x}}$ and can be 
detected in flames using LIF, so a study of a possible state dependence of quenching again plays a dual dynamics/diagnostics role.

The rotational level dependence of quenching of $\widetilde{A}^{2} \mathrm{~A}_{1} \mathrm{NH}_{2}$ by helium was studied at room temperature in a discharge flow. The $\mathrm{NH}_{2}$ was produced by the reaction $\mathrm{F}+\mathrm{NH}_{3}$, and fluorescence excited to three vibrational levels of the A state. The pressure dependence of the time decay of the fluorescence was used to determine the quenching cross sections. The rotational level dependence was found to vary with vibrational level: it increased regularly with $\mathrm{N}^{\prime}$ for $(0,9,0) \Sigma$ but was not significant for the neighboring $(0,8,0)$ and $(0,10,0) \Pi$ levels. This variation is probably not due to collision dynamics as for $\mathrm{NH}$ and $\mathrm{OH}$, because each vibrational level would then behave similarly. The variation is more likely due to mixing with the ground state, which does vary with levels. However, the monotonic increase in quenching from $N^{\prime}=0$ to 7 in $(0,9,0)$ does not correlate with a pattern from known spectroscopic perturbations. The rate constant of $2.5 \times 10^{-11} \mathrm{~cm}^{3} \mathrm{~s}^{-1}$ for $(0,9,0), N^{\prime}=1$ agrees with the results of Dearden, Dixon, and Field. 15

\section{AMMONIA PHOTOLYSIS IN A JET}

During the spring of 1989, D. R. Crosley spent five weeks as a visiting professor at the University of Paris (Orsay), working with P. Brechignac. The experiments conducted in Orsay were supported by the university; the analysis of the data at SRI (now underway) is supported by this project.

The experiment conducted was the 193-nm photolysis of $\mathrm{NH}_{3}$ in a jet expansion with an Ar carrier. The experiment had two goals: (1) the determination of the rotational distribution in the ground electronic state of $\mathrm{NH}$ following photodissociation of cold $\mathrm{NH}_{3}$, and (2) the observation of NH-Ar van der Waals complexes. The data obtained show several features. $\mathrm{NH}$, produced by photolysis before the expansion is complete, becomes rotationally but not vibrationally cold. A distinctly separate rotational distribution in the NH is also observed, with levels populated up to $\mathrm{N}^{\prime \prime}=12$ or above. Other spectroscopic features, not due to $\mathrm{NH}$, are observed in the same spectral region under certain conditions of $\mathrm{NH}_{3} / \mathrm{Ar}$ mixing and pressures and may be due to the complexes.

\section{COLLISIONAL QUENCHING OF NH IN HIGH ROTATIONAL LEVELS}

As noted above, quenching of $\mathrm{NH}$ and $\mathrm{OH}$ can be interpreted in terms of an anisotropic potential surface between the radical and the collider, often governed by attractive forces. An outstanciing remaining question, of importance for both collision 
dynamics and flame diagnostics, is the dependence of the quenching cross section $\sigma_{\mathrm{Q}}$ on $\mathrm{N}^{\prime}$ for high $\mathrm{N}^{\prime}$. For $\mathrm{NH}$, comparisons can be made between low $\mathrm{N}^{\prime}$ and high $\mathrm{N}^{\prime}$ distributions produced directly in the $\mathrm{A}^{3} \Pi_{1}$ state from photodissociation of $\mathrm{NH}_{3}$, indicating a continued decrease above $\mathrm{N}^{*}=7$, but these are not definitive. Our prior LIF studies at room temperature utilized a discharge source, producing only in lower lying rotational levels enough population to furnish adequate signals for the quenching measurements.

The studies at Orsay on photolysis of $\mathrm{NH}_{3}$ in a jet showed that absorption of 193-nm ArF radiation produced $\mathrm{NH}$ radicals in high rotational levels of the ground electronic state. We have now used this method to produce $\mathrm{NH}$ in extremely high $\mathrm{N}^{\prime}$ in bulb experiments. Levels up to and including $N^{\prime}=28$ have been observed in excitation scans. These levels are extremely rotationally energetic; $N^{\prime}=28$ has over $12,000 \mathrm{~cm}^{-1}$ of rotational energy.

Measurements have been made of quenching for both $\mathrm{NH}_{3}$ and $\mathrm{CH}_{4}$ colliders. Quantitative interpretation is complicated by the fact that translational heating of the region irradiated by the ArF laser can produce shock and rarefaction waves, although the results at low $\mathrm{N}^{\prime}$ are in quite reasonable agreement $(20 \%)$ with the room temperature discharge flow results. It is unequivocally clear, however, that the quenching cross section continues to drop with increasing $N^{\prime}$. For $\mathrm{NH}_{3}$, it drops from $92 \AA^{2}$ at $\mathrm{N}^{\prime}=2$ to $35 \AA^{2}$ at $\mathrm{N}^{\prime}=19$ and continues to decrease for higher levels. For $\mathrm{CH}_{4}$, an even larger fractional decrease is found. We had anticipated that $\sigma_{\mathrm{Q}}$ should level off at high $\mathrm{N}^{\prime}$, because the anisotropies in the surface become completely averaged and further rotation would not make any difference. Consequently, very detailed dynamics effects appear to occur in these quenching collisions.

\section{LASER-INDUCED FLUORESCENCE DETECTION OF VINYL RADICALS}

Vinyl radicals, $\mathrm{C}_{2} \mathrm{H}_{3}$, are likely participants in soot formation and the combustion of unsaturated hydrocarbon fuels. It would be extremely valuable to have LIF flame diagnostics of this radical, in addition to our recently developed capabilities for LIF detection ${ }^{16}$ for $\mathrm{CH}$, singlet $\mathrm{CH}_{2}, \mathrm{C}_{2} \mathrm{H}_{2}$, and $\mathrm{HCO}$. Visible absorption has been measured by Hunziker et al. ${ }^{17}$; an origin at $499 \mathrm{~nm}$ is followed by a long progression of $1200 \mathrm{~cm}^{-1}$ to the blue, with a strong band at $423 \mathrm{~nm}$. An absorption coefficient of $30 \mathrm{l} / \mathrm{mol}-\mathrm{cm}$ was measured, indicating facile laser excitation. We thus expected that the molecule should fluoresce well. Theoretical calculations ${ }^{18}$ show that the excited state should be stable against rapid predissociation, and this was confirmed in molecular beam experiments. 19 
A search was made for LIF of $\mathrm{C}_{2} \mathrm{H}_{3}$, using a low pressure discharge flow cell with various chemical schemes for the radical production. Fluorine atoms were reacted with ethylene, hydrogen atoms were added to acetylene, and direct discharges in ethylene and acetylene were examined. Conditions were selected according to current kinetics knowledge to optimize the probable vinyl concentration. Strong LIF signals were observed, but none could be attributed to vinyl. Some time was spent investigating the LIF spectrum of $\mathrm{C}_{3}$ produced in the discharge.

The lack of success is likely due to the thermodynamic and kinetic instability of vinyl in this discharge environment. Therefore we extended our search for vinyl radical LIF, using photolytic production of the radical. The new 193-nm laser, purchased on this project and with SRI International funds, photolyzed butadiene or vinyl chloride. System sensitivity was optimized and calibrated using $\mathrm{NH}_{2}$ LIF following photolysis of ammonia. No vinyl LIF was observed from the strongest absorption bands near $420 \mathrm{~nm}$ to red wavelengths at $610 \mathrm{~nm}$. We can conclude that predissociation lifetimes for the strong bands are less than $10 \mathrm{ps,}$ any band near $610 \mathrm{~nm}$ dissociates on the nanosecond time scale, and the origin of the absorption where predissociation is negligible lies farther to the red and is Franck-Condon inaccessible. (This last conclusion supports Wodtke's recent discussion. ${ }^{20}$ )

\section{REACTION OF HYDROXYL RADICALS WITH ETHYLENE}

RRKM and transition state theory calculations were completed on the $\mathrm{OH}+\mathrm{C}_{2} \mathrm{H}_{4}$ reaction in an attempt to interpret our high temperature laser pyrolysis reaction rate data. At low temperatures the mechanism of this reaction is adduct formation. Our calculations to fit existing data provide $\mathrm{d}$ basis for extrapolating the pressure and temperature dependence of this channel, fix the stability of the adduct at $30 \mathrm{kcal} / \mathrm{mol}$, and indicate that this mechanism is unimportant above $800 \mathrm{~K}$. Four direct high temperature measurements have becis ade recently: our laser pyrolysis experiments, ${ }^{21}$ Tully's photolysis/LIF study, ${ }^{22}$ the pulse radiolysis work of Jonah et al., 23 and a shock tube measurement by Bott and Cohen. ${ }^{24}$ Our measurements at $850 \mathrm{~K}$ and $1200 \mathrm{~K}$ give a rate constant of $2.7 \times 10^{-12} \mathrm{~cm}^{3} \mathrm{~s}^{-1}$. The measurements made by Jonah et al..$^{23}$ and by Bott and Cohen 24 and o: $300 \mathrm{~K}$ measurement are significantly higher than the other measurements. A transition state theory calculation for the expected abstraction reaction to form water and vinyl, where the barrier energy was determined from an Evans-Polanyi correlation with hydrocarbon $\mathrm{C}-\mathrm{H}$ bond energies, was consistent only with the lower set of rate constant values. RRKM calculations were made for possible chemical activation rearrangement channels, but these 
possibilities are incapable of accounting for the larger rate constants. In addition, possible interferences of more complex chemistry in the experimental rate measurements were examined without success. While use of the lower values is recommended, the cause of these differences remains uncertain, and more experiments focussing on real time product measurements are needed.

\section{REACTION OF METHYL RADICALS WITH OXYGEN}

A controversy as to whether the reaction

$$
\mathrm{CH}_{3}+\mathrm{O}_{2} \rightarrow \mathrm{CH}_{2} \mathrm{O}+\mathrm{OH}
$$

can proceed at modest temperatures had long been considered settled but has re-erupted in the recent literature. 25,26 If this reaction proceeds easily, our understanding of the chemical nature of methane and other flames would be radically changed, since the alternative chemical fate of methyl radicals is through their self-reaction to produce higher hydrocarbons, viz:

$$
\mathrm{CH}_{3}+\mathrm{CH}_{3} \rightarrow \mathrm{C}_{2} \mathrm{H}_{5}+\mathrm{H}
$$

or, at low concentrations, their reaction with other radicals such as $\mathrm{O}$ atoms.

We believed that an experiment in these laboratories ${ }^{27}$ using the VLPP technique had shown conclusively that reaction (1) did not occur, but in light of other recent reports we set about applying some of our newer techniques to a different study of this question. We have recently begun a study using our laser pyrolysis capabilities, ${ }^{28}$ along with REMPI spectroscopy of $\mathrm{CH}_{3}$ radicals ${ }^{29}$ and quantitative LIF detection of possible $\mathrm{OH}$ product, 30 to address the possibility of reaction (1).

The experiment is a modification of the LP/LF technique we developed previously during this program. In this experiment an $\mathrm{SF}_{6} /$ bath gas (e.g., Ar, $\mathrm{O}_{2}$ ) mixture is heated with an infrared laser. Unimolecular and bimolecular reactions have been studied. When bimolecular processes are studied, radicals are made by thermal decomposition of a suitable precursor $\left(\mathrm{H}_{2} \mathrm{O}_{2}\right.$ for $\mathrm{OH} ; \mathrm{CH}_{3} \mathrm{~N}_{2} \mathrm{CH}_{3}$ for $\mathrm{CH}_{3}$, etc.). Temperature can be measured by observing various LIF rotational transitions. The reactor has been simply modified by the addition of a pair of circular electrodes. (We now have extensive experience with the REMPI technique from work on other projects. ${ }^{31}$ ) The capability to perform LIF experiments remains, now considerably augmented by the ability to measure nonfluorescing species by using REMPI. Laser pyrolysis of azomethane $\left(\mathrm{CH}_{3} \mathrm{~N}_{2} \mathrm{CH}_{3}\right)$ 
provides the radicals to be detected at $330 \mathrm{~nm}$, which corresponds to a $2+1$ REMPI process, or at $450 \mathrm{~nm}$, which is a $3+1$ process. We are currently characterizing radical spectra and separating the signal due to radicals from those due to shock wave effects and to probe laser ionization of the radical precursors. Thus we should shortly be in a position to follow the decay (or lack thereof) of methyl radical in the presence of oxygen in the temperature range $800-1200 \mathrm{~K}$.

This work was not completed owing to lack of funds. We expect to continue these experiments under the auspices of another sponsor.

\section{HEAT OF FORMATION OF ALKYL FREE RADICALS}

The above subtitle is really a shorthand for what we perceive to be a major problem in gas phase chemical kinetics, with important ramifications for combustion chemistry. There can be no argument that values of the heats of formation of alkyl radicals are in themselves vital for quantitative understanding of the combustion of hydrocarbon fuels, but more far-reaching (see discussion below) is a current discrepancy between results in our laboratory 32 and those reported by others. 33 The specific problem concerns the value and temperature dependence of the rate constant for the reactions between t-butyl radical and $(\mathrm{H} / \mathrm{D}) \mathrm{X}$, where $\mathrm{X}=\mathrm{Br}$ or I. Our value, consistent with previous measurements of the absolute rate constant of similar reactions, is almost two orders of magnitude lower than the recently reported values. In addition, where we find positive activation energies and kinetic isotope effects that would be expected for a simple metathesis reaction, others find negative activation energies.

We have performed some two-channel RRKM calculations ${ }^{61}$ that suggest a potential energy surface containing a bound intermediate of the general class $\mathrm{R} \cdot \mathrm{XH}$ compatible with such a negative activation energy. (This also relates, interestingly, to current DOE-sponsored studies of van der Waals complexes. ${ }^{39}$ ) However, this model requires an intermediate bound by $8-10 \mathrm{kcal} / \mathrm{mol}$ and an inverse isotope effect. An inverse isotope effect is the opposite of the few existing observations, and such a stable complex is unexpected. Clearly a careful study of the isotope effect and temperature dependence is warranted as a search for evidence of intermediates of this type.

The reason this controversy is of such general importance is that it may suggest fundamental problems with experimental techniques that have been used to obtain a good deal of the data used in current combustion and atmospheric chemistry. Gutman and coworkers have used the same rechnique that yields the negative activation energies for the 
$\mathrm{R}+\mathrm{HX}$ processes to examine many $\mathrm{R}+\mathrm{O}_{2}$ reactions. ${ }^{35}$ These reactions are the key to our understanding of ignition, cool flames, and product branching ratios in the oxidation of hydrocarbons. Gutman et al. have suggested values for the thermochemistry of the ensuing peroxy radicals that are different than those used previously. On the other hand, we have produced large numbers of heats of formation by the halogenation technique, 36 which heats depend on the value for the activation energy of $R+H X$ reactions being small and positive. We have also measured ${ }^{37}$ many radical molecule rate constants by using the technique that we call VLPФ, which was used in the t-butyl + (H/D)X studies.

In several instances we have felt it important to comment on radical thermochemistry results suggested by other workers. This is reflected in publications 14 and 15 in our list of journal publications. The first report concerns basic errors in deriving $\mathrm{H}_{x} \mathrm{O}_{y}$ thermochemistry from electrochemical potentials as done in work reported in a recent publication. The second note corrects literature values for cyclopentadienyl radical enthalpies and organometallic sandwich compounds. This interesting 5- $\pi$ electron ring system has recently been implicated in the autocatalysis of methane pyrolysis. ${ }^{38}$

\section{TRANSITION STATE THEORY-BASED RATE CONSTANT ESTIMATIONS}

In concert with the thought that complex chemical systems such as combustion can only be understood through a model, and given the practical impossibility of measuring all processes under conditions of interest, we continue to apply simple transition state theory (TST) to the initial estimation of many rate processes. This method is particularly applicable to simple metathesis reactions, and we have analyzed many of the reactions of this type in the methane flame model. Results are given in Table 1.

Our method, illustrated in a paper in a recent Combustion Symposium, ${ }^{39}$ uses techniques described in Benson's Thermochemical Kinetics 40 to estimate transition state properties associated with the transition state entropy. Where available, we use measured values of the rate constants to establish the enthalpy of the transition state; otherwise we make estimates by analogy.

We have recently applied related TST approaches to band scission reactions to describe the proper way to derive bond energies from pyrolysis activation energies. Care must be taken as this relationship is not simple and straightforward. This collaboration with a sabbatical visitor and renowned expert on thermochemical kinetics, Professor Alan Rodgers of Texas A\&M University, is being prepared for publication. 


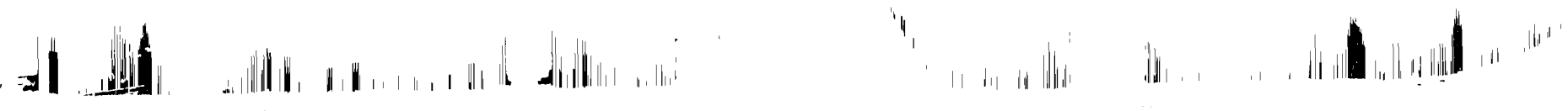
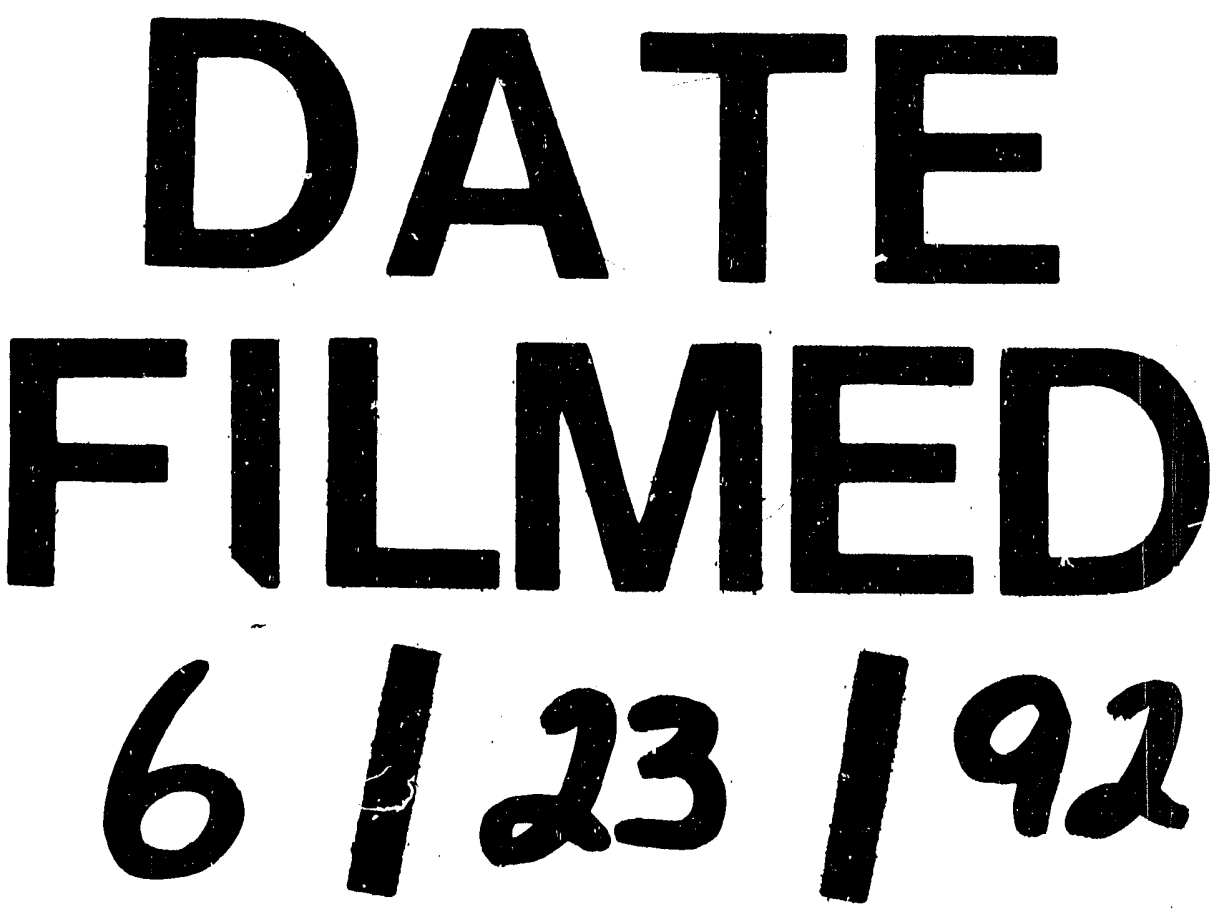
